\title{
Negative Allosteric Modulators Selective for The NR2B Subtype of The NMDA Receptor Impair Cognition in Multiple Domains
}

\author{
Michael R Weed", , Mark Bookbinder', Joseph Polino', Deborah Keavy', Rudolf N Cardinal ${ }^{2,3}$, \\ Jean Simmermacher-Mayer ${ }^{4}$, Fu-ni L Cometa ${ }^{4}$, Dalton King ${ }^{5}$, Srinivasan Thangathirupathy ${ }^{6}$, John E Macor ${ }^{5}$ \\ and Linda J Bristow' \\ 'Genetically Defined Diseases and Genomics, Bristol-Myers Squibb Company, Wallingford, CT, USA; ${ }^{2}$ Behavioral and Clinical Neuroscience Institute, \\ Department of Psychiatry, University of Cambridge, Cambridge, UK; ${ }^{3}$ Cambridgeshire and Peterborough NHS Foundation Trust, Cambridge, UK; \\ ${ }^{4}$ Pharmaceutical Candidate Optimization, Bristol-Myers Squibb Company, Wallingford, CT, USA; ${ }^{5}$ Discovery Chemistry, Bristol-Myers Squibb \\ Company, Wallingford, CT, USA; ${ }^{6}$ Discovery Chemistry, Biocon Bristol-Myers Squibb Research \& Development Center, Bangalore, India
}

Antidepressant activity of N-methyl-D-aspartate (NMDA) receptor antagonists and negative allosteric modulators (NAMs) has led to increased investigation of their behavioral pharmacology. NMDA antagonists, such as ketamine, impair cognition in multiple species and in multiple cognitive domains. However, studies with NR2B subtype-selective NAMs have reported mixed results in rodents including increased impulsivity, no effect on cognition, impairment or even improvement of some cognitive tasks. To date, the effects of NR2Bselective NAMs on cognitive tests have not been reported in nonhuman primates. The current study evaluated two selective NR2B NAMs, CPI0I,606 and BMT-108908, along with the nonselective NMDA antagonists, ketamine and AZD6765, in the nonhuman primate Cambridge Neuropsychological Test Automated Battery (CANTAB) list-based delayed match to sample (list-DMS) task. Ketamine and the two NMDA NR2B NAMs produced selective impairments in memory in the list-DMS task. AZD6765 impaired performance in a nonspecific manner. In a separate cohort, CPI0I,606 impaired performance of the nonhuman primate CANTAB visuo-spatial Paired Associates Learning (vsPAL) task with a selective impairment at more difficult conditions. The results of these studies clearly show that systemic administration of a selective NR2B NAM can cause transient cognitive impairment in multiple cognitive domains.

Neuropsychopharmacology (20 I6) 4I, 568-577; doi:I0.I038/npp.20 I5. I84; published online I5 July 20 I 5

\section{INTRODUCTION}

The potential for $\mathrm{N}$-methyl-D-aspartate (NMDA) receptor antagonists, such as ketamine, to function as antidepressants with rapid clinical onset has led to increased research on their behavioral pharmacology. Clinical studies in small numbers of patients have repeatedly shown that a single, intravenous dose of ketamine can produce an antidepressant effect that lasts for several days in treatment-resistant, depressed patients (Abdallah et al, 2015; Coyle and Laws, 2015). However, like most nonselective NMDA receptor channel blockers, ketamine has a number of adverse effects including dissociative effects and cognitive impairment, leading to the search for novel agents that are better tolerated (Morgan and Curran, 2006; Newcomer and Krystal, 2001). One approach is the development of NMDA receptor

*Correspondence: Dr MR Weed, Genetically Defined Diseases and Genomics, Bristol-Myers Squibb Company, 5 Research Parkway, Wallingford, CT 06492, USA, Tel: +I 860203677 65|4, Fax: + I 860203677 7569, E-mail: michael.weed@bms.com

Received 3 March 2015; revised 28 May 2015; accepted II June 20I5; accepted article preview online 24 June 2015 channel blockers with low potential to become trapped in the channel pore following repetitive channel activation as exemplified by AZD6765 (lanicemine). Although this agent is still a nonselective NMDA receptor antagonist, AZD6765 shows 'low trapping' in electrophysiology studies and appears to be well tolerated in humans (Sanacora et al, 2014; Zarate et al, 2013). Unfortunately, the antidepressant efficacy of this agent is now uncertain given that the beneficial effects seen in early clinical testing were not observed in a larger clinical trial (Sanacora and Schatzberg, 2015). A second approach is the development of subtype-selective NMDA receptor antagonists with attention focused on the NR2B subtype. CP101,606 (traxoprodil) is a negative allosteric modulator (NAM), which binds to a site present on the N-terminal domain of the NR2B receptor and prevents channel activation (Menniti et al, 1998). In a small, proof of concept, clinical trial a single IV infusion of CP101,606 produced an antidepressant effect at a dose with low potential to produce dissociative effects in patients (Preskorn et al, 2008).

Despite renewed interest in the antidepressant potential of NMDA receptor antagonists, the clinical development of these agents has historically been challenging, and the ability 
to understand efficacy and tolerability in preclinical species is important. With respect to cognitive impairment, numerous preclinical studies have shown that ketamine impairs cognition in rodents and nonhuman primates, effects similar to those reported in humans (Buccafusco and Terry, 2009; Neill et al, 2010; Roberts et al, 2010; Taffe et al, 2002a). In contrast, the effects of NR2B NAMs have been less well studied and have produced mixed results. Unlike NMDA channel blockers such as MK-801 or phencyclidine systemic administration of NR2B NAMs in rats does not adversely affect spatial memory in operant-delayed match to position tasks (Higgins et al, 2003; Smith et al, 2011). Similar results were also seen in the Morris watermaze task where systemic NR2B NAM treatment had no effect on memory acquisition or retention for the location of the hidden platform (Guscott et al, 2003; Higgins et al, 2005; Higgins et al, 2003; Okiyama et al, 1997). Importantly, evaluation of NMDA receptor occupancy in tissues collected from experimental subjects showed that $\sim 100 \%$ NR2B occupancy was achieved in hippocampus and cortex after CP101,606 dosing, suggesting that inadequate dosing or lack of receptor occupancy does not explain the lack of memory impairment (Guscott et al, 2003). On the other hand, direct infusion of the NR2B NAM Ro25-6981 into the rat hippocampus impaired spatial working memory assessed using a watermaze or $\mathrm{T}$ maze delayed alternation paradigm, suggesting that local versus systemic dosing can lead to different effects (Zhang et al, 2013). Mixed results have also been reported in mice with systemic dosing of the NR2B NAM Ro25-6981 producing both an impairment of spatial working memory ( $\mathrm{Y}$ maze spontaneous alternation task) and an improvement of executive function (attentional set shifting) (Hanson et al, 2013; Kos et al, 2011). Thus, NR2B NAMs produce very diverse effects on cognition in rodents depending more on factors such as route of delivery, the cognitive domain being assessed and the various tasks explored than on levels of NR2B receptor occupancy.

Although several studies have explored NR2B NAM treatment in rodents, the effect of NR2B NAM treatment on cognition in humans and nonhuman primates has not been adequately explored. In humans, the effects reported are largely descriptive and include anecdotal reports of impaired delayed word recall memory in healthy humans and abnormal thinking (including dissociation and amnesia) in Parkinson's Disease patients following IV infusion of CP101,606 (Merchant et al, 1999; Nutt et al, 2008). Unfortunately, the effect of CP101,606 infusion on cognition in patients with treatment-resistant depression was not reported. The therapeutic margin between antidepressant effect and cognitive impairment is therefore not well established. In nonhuman primates there are no published reports describing the impact of systemic NR2B NAM treatment on cognition. Local injection of Ro25-6981 into the dorsolateral prefrontal cortex (dlPFC) of macaques attenuates firing of neurons active during the delay period of a working memory task; however, the impact on task accuracy was not reported and systemic dosing of an NR2B NAM was not examined (Wang et al, 2013). Thus, although these electrophysiological results suggest that NR2B NAMs may impair working memory in nonhuman primates, direct demonstration of cognitive impairment has not yet been shown.
The present studies assessed the acute effects of NMDA antagonist treatment on cognitive performance in cynomolgus monkeys. To optimize the translational value of these studies, two tests from the nonhuman primate Cambridge Neuropsychological Test Automated Battery (CANTAB) battery were used: a list-based variant of the delayed match to sample (DMS) task and the visuo-spatial paired associates learning (vsPAL) test. Ketamine and the 'low trapping' nonselective NMDA channel blocker, AZD6765, were chosen for comparison with the selective NR2B NAMs CP101,606 and BMT-108908. BMT-108908 is a novel agent, which potently displaces $\left[{ }^{3} \mathrm{H}\right] \mathrm{Ro} 25-6981$ binding to native human, cynomoglus monkey, and rat brain NR2B receptors ( $\mathrm{Ki}=1.6,0.71$, and $1.4 \mathrm{nM}$, respectively). In xenopus oocytes expressing recombinant human NMDA receptors, BMT-108908 selectively inhibits hNR1A/2B receptor function $(\mathrm{IC} 50=4.2 \mathrm{nM}$ ) and is inactive at the NR2A, NR2C, and NR2D receptor subtypes. In addition, BMT-108908 has no significant activity at 43 relevant CNS receptors and/or enzymes (unpublished data). The detailed pharmacological profile of BMT-108908 will be presented elsewhere.

\section{MATERIALS AND METHODS}

\section{Subjects}

The cognitive studies used 18 male cynomolgus monkeys (Macaca Fasicularis). Each had been used in prior pharmacological studies, and at least a month separated studies. The monkeys were 5-7 years of age and weighed $5.0-8.5 \mathrm{~kg}$. Monkeys were typically pair-housed and fed standard monkey chow (Harlan Teklad Global 20\% protein Primate Diet 2050) in sufficient quantities to ensure normal growth while maintaining motivation to perform the tasks (Weed et al, 1999).

The pharmacokinetic studies used six male cynomolgus monkeys that had been previously implanted with vascular access ports in a femoral artery and a femoral vein. Housing and care were as described above; however, these animals were not food restricted.

For all animals, water was continuously available except during studies, and fresh fruit or dietary enrichment was provided twice weekly. Toys and foraging devices were routinely provided and television programs were available in the monkey colony rooms. Subjects were fitted with plastic or metal neck collars (Primate Products, Immokalee, FL). Laboratory animal care was according to Public Health Service Policy on the Humane Care and Use of Laboratory Animals, and the Guide for the Care and use of Laboratory Animals (NRC, 2011). The protocol was approved by the Wallingford Animal Care and Use Committee of the BristolMyers Squibb Company.

\section{Apparatus}

For behavioral testing, monkeys were seated comfortably in nonhuman primate chairs (Primate Products), and placed in a sound-attenuated chamber (Med-Associates, St Albans, VT) with a touch-sensitive computer monitor within easy reach. Session events were controlled by the nonhuman primate CANTAB software; (Lafayette Instruments, Lafayette, IN) running in Whisker Control (Cardinal and Aitken, 

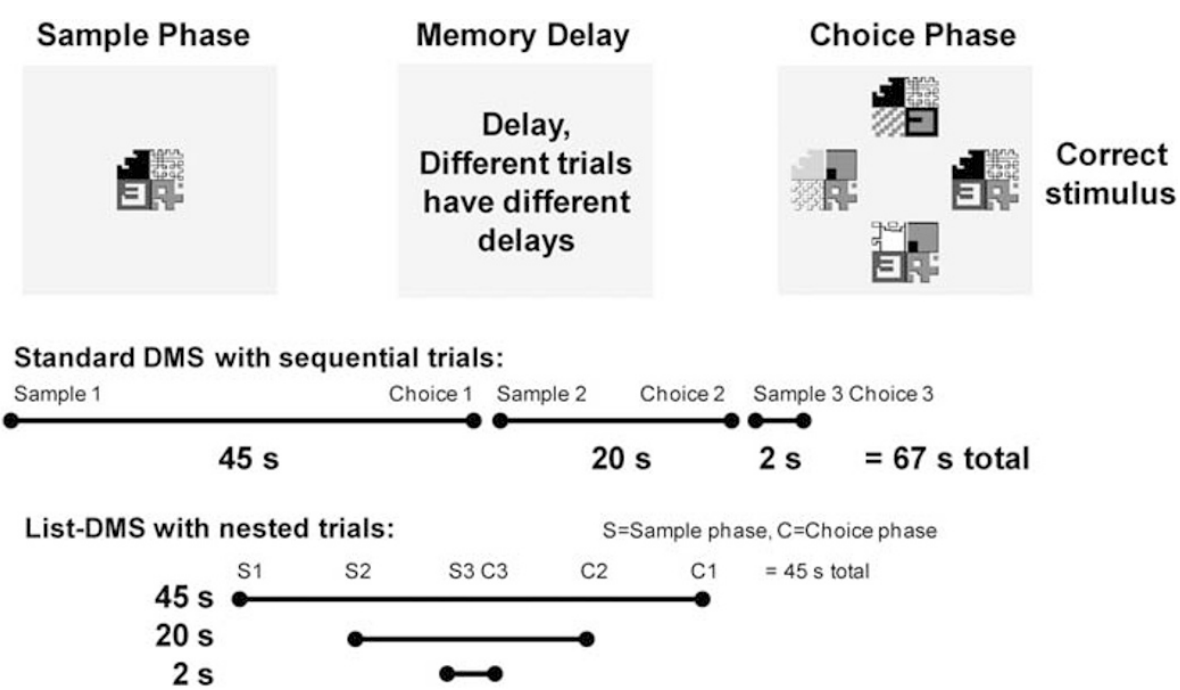

Figure I Schematic of the DMS procedure. Actual stimuli are in color and each quadrant of the stimulus is a uniform color. Distracters in the choice phase may mimic the shape of any quadrant of the sample stimulus and use the same or different color. The list-DMS protocol shortens the time required to perform the test, which can be important for compounds with shorter half lives.

2010). Following correct responses, a dispenser delivered $190 \mathrm{mg}$ banana-flavored pellets (Bioserv, Frenchtown, PA).

\section{Behavioral Procedures: List-DMS}

Animals were first trained to proficiency on the standard nonhuman primate CANTAB DMS where an abstract stimulus was presented in the center of the screen for the monkey to touch ('sample phase') and, after a memory delay, the sample stimulus and three distracter stimuli were presented in the 'choice phase' (total of four stimuli in each corner of screen).

List-DMS differs from standard DMS in that three sample stimuli were presented sequentially, prior to any choices (Figure 1). The choice phases for the respective sample stimuli also occurred sequentially, after their respective memory delays. The three trials are thus 'nested' within the longest memory delay, reducing the session length, which may be important for testing short acting compounds.

For this study the memory delays were 2, 20, and $45 \mathrm{~s}$. The sequence of events was: sample stimulus A (which would have a 45-s memory delay) was presented and touched, resulting in a screen blank. Next, sample stimulus B (which would have a 20 -s memory delay), was presented and touched, resulting in another screen blank. Sample stimulus $\mathrm{C}$ (which would have a 2-s delay) was touched, resulting in a screen blank for the 2-s memory delay, after which the choice stimuli for stimulus $\mathrm{C}$ were presented. After a response, pellets were delivered (for a correct response) or not (for an error), and the screen was blanked until the 20-s memory delay for stimulus B expired. The choice phase for stimulus B proceeded as for stimulus $\mathrm{C}$, followed by a screen blank until the 45-s memory delay from stimulus A expired. Following the end of the choice phase for stimulus A there was a 10-s screen blank before the process repeated with all new stimuli.

In either the sample or choice phase, the monkey had $5 \mathrm{~s}$ to respond to the stimulus, or the trial was considered an 'omission'. If the omission was in the sample phase, the choice phase for that sample stimulus was not presented, but the timing of sample and choice phases for other stimuli remain unchanged. Three distracter stimuli were used for all delays, and therefore, chance responding would yield $25 \%$ correct in this procedure. A session consisted of 20 lists, allowing for 60 total trials.

\section{Behavioral Procedures: vsPAL}

In brief, the monkey was required to learn and remember the association of a visual stimulus and a location on the touch screen (Supplementary Figure 1; Taffe et al, 2002b). For a given trial there were either two, three, or four stimulus/ location pairs to remember. Sample stimuli were presented in a given location in a list sequence, with $1 \mathrm{~s}$ between stimuli. After all stimuli were sampled in their appropriate position, there was a 15-s memory delay. In the choice phase, a stimulus was presented in the correct location as well as three incorrect locations. Positions were randomly selected from nine possible locations in a $3 \times 3$ grid. Each touch in the correct location resulted in food pellet delivery. If the animal failed to respond within $30 \mathrm{~s}$, or if the animal responded in an incorrect location, the trial stopped there. After a 10-s time out, the same sequence of stimuli in the same locations repeated, starting with the sample phase. The animal had the initial attempt plus up to five repetitions to learn the correct stimulus-location pairs. If the animal responded to all stimuli correctly on the initial presentation, the trial was scored as 'initial correct'. If a trial was completed correctly after an initial error, the trial was scored as correct eventually ('eventual correct'). Stimuli changed after correct trials or after six failed attempts. As with previous studies, a session consisted of 10 trials at each difficulty level presented in an ascending order of difficulty (Taffe et al, 2002b).

\section{Drugs}

Racemic ketamine $\mathrm{HCl}$ was purchased as a $10 \mathrm{mg} / \mathrm{kg}$ solution (Ketaset, Fort Dodge Animal Health, Fort Dodge, IA). CP101,606 (traxoprodil, methanesulfonic acid salt, vehicle: $5 \%$ DMSO/10\% propylene glycol/15\% methylcellulose/70\% sterile water), AZD6765 (lanicemine, $\mathrm{HCl}$, vehicle: 5\% 
Table I Summary of Details from RM ANOVAs on List-DMS Performance

\% Correct, dose-response function

\begin{tabular}{|c|c|c|c|c|c|}
\hline & Difficulty & Treatment & Interaction & Response latency & $\%$ Task completion \\
\hline Ketamine & $F_{2,16}=42.3 ; p<0.0001$ & $F_{4,32}=12.4 ; p<0.0001$ & $F_{8,64}=1.4 ; p>0.05$ & $F_{4,32}=5.9 ; p=0.001$ & $F_{4,32}=6.2 ; p=0.0008$ \\
\hline AZD6765 & $F_{2,14}=35.9 ; p<0.0001$ & $F_{3,21}=12.4 ; p<0.000 \mid$ & $F_{6,42}=12.5 ; p<0.000 \mid$ & $F_{3,21}=0.2 ; p>0.05$ & $F_{3,21}=4.9 ; p=0.01$ \\
\hline BMT-I08908 & $F_{2,16}=211.6 ; p<0.0001$ & $F_{4,32}=39.5 ; p<0.0001$ & $F_{8.64}=12.8 ; p<0.0001$ & $F_{4,32}=4.4 ; p=0.006$ & $F_{4,32}=0.9 ; p>0.05$ \\
\hline
\end{tabular}

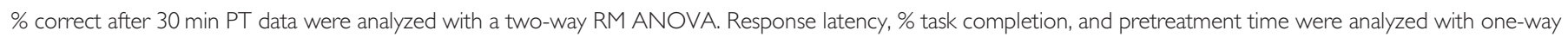
RM ANOVAs.

DMSO/95\% sterile water), and BMT-108908 (free base, vehicle: $30 \%$ hydroxypropyl beta cyclodextrin $/ 70 \%$ citrate buffer, $\mathrm{pH}$ 4.0) were synthesized at Bristol-Myers Squibb. Compounds were administered intramuscularly at $0.1-$ $0.2 \mathrm{ml} / \mathrm{kg}$. Doses refer to the free base.

\section{Drug Testing}

All compounds were tested in the list-DMS procedure. Monkeys were included in dose-response analyses if they achieved stable baseline performance $( \pm 15 \%$ over the week prior to testing). If an animal's percent trials completed dropped below $20 \%$ for a given test session, their accuracy and latency data were discarded and interpolated with the group mean for the repeated-measures analysis of variance (RM-ANOVA). Ketamine was administered $15 \mathrm{~min}$ before the session and all other compounds were administered $30 \mathrm{~min}$ prior to the session. To determine whether cognitive impairment of CP101,606 was specific to the list-DMS task, CP101,606 was also tested in the vsPAL procedure. Determinations of performance after vehicle occurred prior to, during, and at the end of the dose-response function for each drug (minimum of three vehicle determinations for a given drug). Drugs were tested twice weekly with baseline performance sessions on days in between. On test days the cohort was divided roughly in half, and one-half received one dose of the drug and the other half received another dose of the drug. Drug doses were thus administered in a mixed order.

To determine exposure-effect relationships for cognitive impairment of CP101,606 and BMT-108908, the effects of both compounds were also studied after 3 and $5 \mathrm{~h}$ pretreatment (in addition to the $0.5 \mathrm{~h}$ pretreatment used in the dose-response function). Blood samples were obtained immediately following the behavioral session at these times. Vehicle pretreatments at 3 and $5 \mathrm{~h}$ were determined for each compound, and comparisons were made between the mean of the respective drug and vehicles. In addition, for the longdelay condition, the difference between performance after vehicle and drug was calculated and related to the exposure.

\section{Statistical Analysis}

Performance accuracy (\% correct (list-DMS); \% initially and eventually correct (vsPAL)) was analyzed with RM-ANOVA with the two within-subject factors of difficulty (memory delay or number of stimuli per trial) and treatment. Omissions were not included in the percent correct measure,
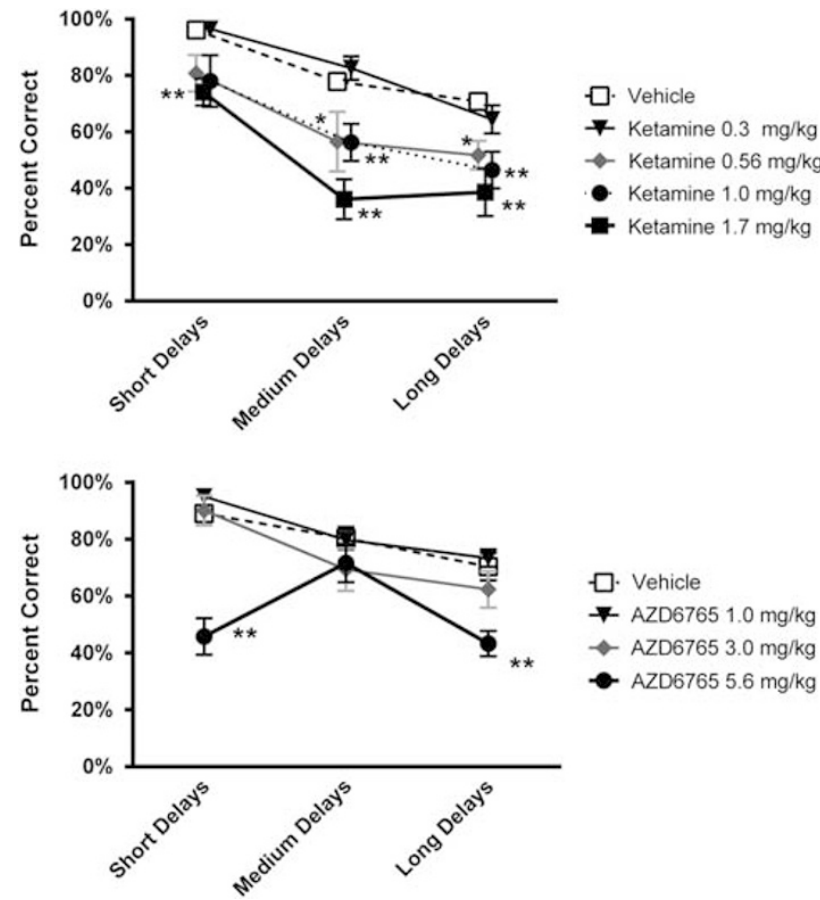

Figure 2 Effect of acute treatment with the NMDA channel blockers RSketamine (top, $N=9$ ) and AZD6765 (bottom, $N=8$ ) on list-DMS performance in cynomolgus monkeys. Results are presented as the group mean $\pm \mathrm{SEM}$; $*$ and $* *$ indicate that performance differs from vehicle treatment at the $p<0.05$ and $p<0.0$ I levels, respectively.

but contributed to percent task completed. Percent task completed was the proportion of trials on which the animal responded (in both sample and choice phase) divided by the total number of trials (ie, \# responded/(\# responded+\# omitted)). Latencies to respond in the choice phase (averaged across delays) and percent task completed for the entire session were analyzed with a one-way RM-ANOVA. HolmSidak's method was used for post hoc comparisons against vehicle. Statistical tests were performed in Prism v6.05 (Graphpad, San Diego, CA) and presented in detail in Tables 1 and 4 .

\section{RESULTS}

\section{List-DMS Performance}

Acute treatment with ketamine impaired performance on list-DMS in a dose- and difficulty-dependent fashion 
Table 2 Group Means for Overall Response Latency in List-DMS in Milliseconds

\begin{tabular}{|c|c|c|c|c|}
\hline & Ketamine (ms) & AZD6765 (ms) & CPIOI,606 (ms) & ВMT-I $08908(\mathrm{~ms})$ \\
\hline Vehicle (SEM) & $2405(82.4)$ & $2459(72.0)$ & $2526(70.7)$ & $2443(80.3)$ \\
\hline $0.10 \mathrm{mg} / \mathrm{kg}$ (SEM) & n.t. & n.t. & n.t. & $2381(171.6)$ \\
\hline $0.30 \mathrm{mg} / \mathrm{kg}$ (SEM) & $250 \mid(\mid 37.2)$ & n.t. & $2382(135.7)$ & $2574(82.8)$ \\
\hline $0.56 \mathrm{mg} / \mathrm{kg}$ (SEM) & $2521(192.7)$ & n.t. & n.t. & n.t. \\
\hline 1.00 mg/kg (SEM) & $2386(215.1)$ & $2460(107.3)$ & $2604(107.5)$ & $2798(65.42)^{*}$ \\
\hline 3.00 mg/kg (SEM) & n.t. & $2563(140.2)$ & $2557(122.8)$ & $2744(94.78)$ \\
\hline 5.60 mg/kg (SEM) & n.t. & $2456(215.3)$ & $243 \mid(\mid 42.7)$ & n.t. \\
\hline 10.00 mg/kg (SEM) & n.t. & n.t. & $2802(98.7)$ & n.t. \\
\hline
\end{tabular}

Abbreviation: n.t., not tested. * and *** indicate performance differed from vehicle at $p<0.05$ and $p<0.0$ I levels, respectively, using Holm-Sidak test.

Table 3 Group Means for List-DMS Percent Completion

\begin{tabular}{|c|c|c|c|c|}
\hline Vehicle (SEM) & $98.4 \%(0.5 \%)$ & $97.0 \%(0.7 \%)$ & $96.9 \%(1.1 \%)$ & $96.4 \%(1.7 \%)$ \\
\hline 0.10 mg/kg (SEM) & n.t. & n.t. & n.t. & $94.8 \%(4.7 \%)$ \\
\hline 0.30 mg/kg (SEM) & $75.4 \%(12.8 \%)$ & n.t. & $95.8 \%(1.8 \%)$ & $96.2 \%(2.4 \%)$ \\
\hline 0.56 mg/kg (SEM) & $74.0 \%(8.2 \%)$ & n.t. & n.t. & n.t. \\
\hline 1.70 mg/kg (SEM) & $43.2 \%(12.5 \%)^{* * *}$ & n.t. & n.t. & n.t. \\
\hline 3.00 mg/kg (SEM) & n.t. & $94.1 \%(3.3 \%)$ & $83.7 \%(7.8 \%)$ & $90.9 \%(6.8 \%)$ \\
\hline 5.60 mg/kg (SEM) & n.t. & $55.8 \%(\mid 4.2 \%)^{* * *}$ & $66.7 \%(12.3 \%)^{*}$ & n.t. \\
\hline 10.00 mg/kg (SEM) & n.t. & n.t. & $70.4 \%$ (9.2\%) & n.t. \\
\hline
\end{tabular}

Abbreviation: n.t., not tested. * and *** indicate performance differed from vehicle at $p<0.05$ and $p<0.0$ I levels, respectively, using Holm-Sidak test.

(Figure 2, top; Table 1 and Supplementary Table S1). RMANOVA (Table 1) and post hoc tests confirmed that impairment was dose-dependent as $0.56 \mathrm{mg} / \mathrm{kg}$, and higher doses impaired accuracy, whereas $0.3 \mathrm{mg} / \mathrm{kg}$ did not differ significantly from vehicle (Figure 2, top; Supplementary Table S1). Ketamine's effects were difficulty dependent for the 0.56 and $1.0 \mathrm{mg} / \mathrm{kg}$ doses as accuracy at short delays did not differ from vehicle, whereas accuracy was significantly impaired at medium and long delays. Latency to respond and percent task completion were not impaired following $0.56 \mathrm{mg} / \mathrm{kg}$ ketamine, consistent with a specific reduction in memory at this dose (Tables 1, 2, and 3). Ketamine decreased percent task completed at 1.0 and $1.7 \mathrm{mg} / \mathrm{kg}$. After $1.7 \mathrm{mg} / \mathrm{kg}$ ketamine list-DMS performance was impaired on all measures, the contribution of memory impairment relative to motivational or psychomotor impairment could not be determined for this dose. Average plasma ketamine concentrations measured $15 \mathrm{~min}$ after dosing with 0.56 and $1.0 \mathrm{mg} / \mathrm{kg}$ were 595 and $705 \mathrm{nM}$, respectively (Supplementary Table S2).

Acute treatment with AZD6765 had no effect on performance accuracy at any delay condition at doses of 1 and $3 \mathrm{mg} / \mathrm{kg}$ i.m. (Figure 2, bottom; Table 1 and Supplementary Table S1). In addition, latency to respond and \% task completed were also not affected at these doses (Tables 2 and 3 ). In contrast, a significant decrease in accuracy was observed at both the short- and long-delay conditions in subjects treated with $5.6 \mathrm{mg} / \mathrm{kg}$ AZD6765. A significant reduction in $\%$ task completed was also observed at this dose, suggesting that non-specific effects may account for the apparent memory impairment. Higher doses of AZD6765 could not be examined, as prior studies showed severe emesis in animals treated with $10 \mathrm{mg} / \mathrm{kg}$ AZD6765. The average plasma concentration determined $30 \mathrm{~min}$ after dosing with $5.6 \mathrm{mg} / \mathrm{kg}$ AZD6765 was $7168 \mathrm{nM}$ (Supplementary Table S3).

Acute treatment with CP101,606 produced a dose- and difficulty-dependent impairment in performance accuracy in the list-DMS task. At doses $\geqslant 1 \mathrm{mg} / \mathrm{kg}$, a selective impairment at the medium- and long-delay conditions was observed, whereas accuracy at short delays was not affected (Figure 3, top; Table 1 and Supplementary Table S1). CP101,606 did not impair response latency at any dose tested (Table 2); however, a modest, but significant, reduction in \% task completed was observed at the $5.6 \mathrm{mg}$ / $\mathrm{kg}$ dose (Table 3). Doses of 1.0 and $3.0 \mathrm{mg} / \mathrm{kg}$ impaired accuracy without significantly affecting response latency or percent task completed, consistent with a selective disruption of memory at medium and long delays. The average plasma concentration measured $30 \mathrm{~min}$ after dosing with $1.0 \mathrm{mg} / \mathrm{kg}$ or $3.0 \mathrm{mg} / \mathrm{kg}$ CP101,606 was 471 and $1137 \mathrm{nM}$, respectively (Supplementary Table S4). 
Figure 4, top left panel illustrates the time course of impairment of list-DMS performance following $1.0 \mathrm{mg} / \mathrm{kg}$ $\mathrm{CP} 101,606$ as represented by \% correct in the long-delay condition (RM-ANOVA of time course: $\mathrm{F}_{3.24}=15.6$; $p<0.0001)$. Although performance was significantly
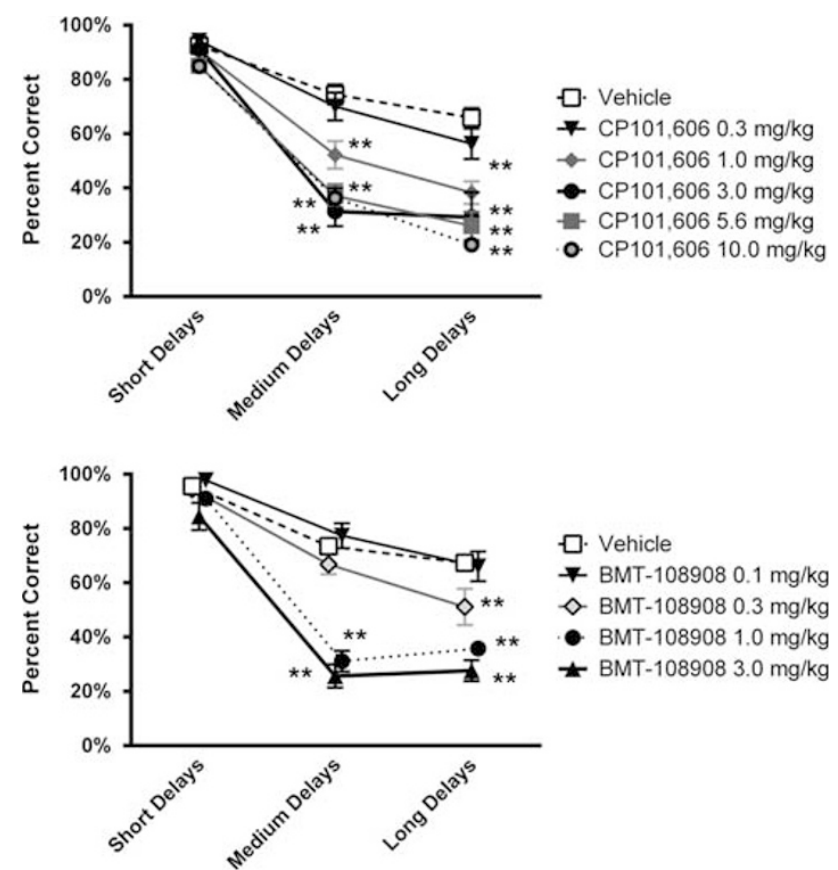

Figure 3 Effect of acute treatment with the NR2B-selective NAMs CPI0I,606 (top, $N=9$ ) and BMT-I08908 (bottom, $N=9$ ) on list-DMS performance in cynomolgus monkeys. Results are expressed as the group mean $\pm \mathrm{SEM}$; $*$ and $* *$ indicate that performance differs from vehicle treatment at the $p<0.05$ and $p<0.0$ I levels, respectively. impaired $30 \mathrm{~min}$ after dosing, the effect was no longer significant at $3 \mathrm{~h}(p=0.09$ from Holm-Sidak test) and returned to vehicle-control levels by $5 \mathrm{~h}$ post dose. Recovery from impairment was closely related to plasma CP101,606 concentrations and cognitive impairment was consistently observed at plasma concentrations $\geqslant 471 \mathrm{nM}$ (Figure 4, top right panel; Supplementary Table S4).

Acute treatment with BMT-108908 also produced a doseand difficulty-dependent impairment in performance accuracy in the list-DMS. At doses $\geqslant 0.3 \mathrm{mg} / \mathrm{kg}$ a selective impairment in the medium- and/or long-delay condition was observed, whereas accuracy in the short delay condition was not altered (Figure 3, bottom; Table 1 and Supplementary Table S1). BMT-108908 did not impair \% task completion at any dose tested; however, a significant increase in the average latency to respond of $355 \mathrm{~ms}$ was observed at the $1.0 \mathrm{mg} / \mathrm{kg}$ dose (Tables 2 and 3). Performance after $0.3 \mathrm{mg} / \mathrm{kg}$ BMT-108908 was consistent with a selective impairment in memory at the long-delay condition. Average plasma concentrations of BMT-108908, $30 \mathrm{~min}$ after dosing with $0.3 \mathrm{mg} / \mathrm{kg}$, were $279 \mathrm{nM}$ (Supplementary Table S5).

Figure 4, bottom left panel illustrates the time course of impairment of list-DMS performance following $1.0 \mathrm{mg} / \mathrm{kg}$ BMT-108908 as represented by \% correct at the long-delay condition. With $30 \mathrm{~min}$ and $3 \mathrm{~h}$ pretreatment times there was a significant impairment, with full recovery to vehicle-treated control levels by $5 \mathrm{~h}$ post dose (RM ANOVA of time course: $\left.\mathrm{F}_{3.24}=8.8 ; p=0.0004\right)$. Recovery from impairment was closely related to plasma BMT-108908 concentrations and cognitive impairment was consistently observed at plasma concentrations $\geqslant 275 \mathrm{nM}$ (Figure 4, bottom right panel; Supplementary Table S5).
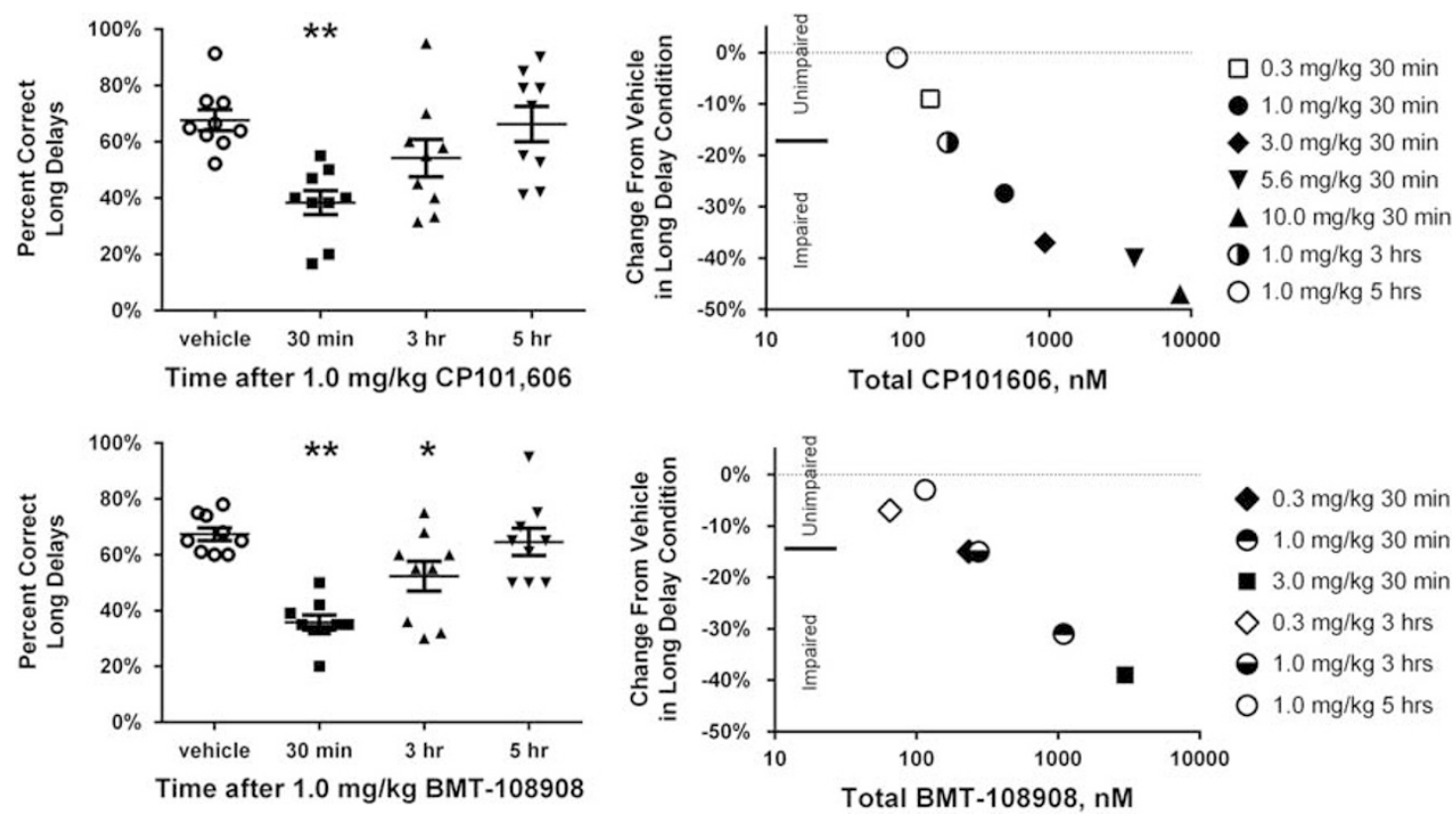

Figure 4 Time course of list-DMS impairment by CPI0I,606 and BMT-108908 (left panels) and plasma exposure vs impairment relationship for CPI0I,606 and BMT-I08908 (right panels). Left panels: $y$ axis is percent correct responding for long delays after I mg/kg CPI0I,606 (top left) and I mg/kg BMT- 108908 (bottom left). Bars are SEM of group and line is group mean. * and ** indicate that performance differs from performance after vehicle at the $p<0.05$ and $p<0.0$ I levels, respectively. Right panels: mean \% change from vehicle at long delay is plotted against average plasma concentration of total compound for CPI0I,606 (top) and BMT-I08908 (bottom). Filled or half-filled symbols indicate significantly impaired list-DMS performance. 


\section{vsPAL Performance}

Acute treatment with CP101,606 produced a dose- and difficulty-dependent impairment of initial accuracy in the vsPAL task (Figure 5, top; Table 4, Supplementary Table S6). This impairment was only observed at the most difficult four-stimuli condition at $0.3 \mathrm{mg} / \mathrm{kg} \mathrm{CP} 101,606$ but was apparent on both the three- and four-stimuli conditions after $1.0 \mathrm{mg} / \mathrm{kg}$. After $3.0 \mathrm{mg} / \mathrm{kg}$, performance was impaired at all difficulty levels. In addition, CP101,606 impaired eventual accuracy in the four stimulus condition after the $1.0 \mathrm{mg} / \mathrm{kg}$ dose (Figure 5, bottom; Table 4 and Supplementary Table S6). After $3 \mathrm{mg} / \mathrm{kg}$, eventual accuracy was impaired at all difficulty levels and significant impairments in response latency and \% trials completed were also observed (Table 5). The results suggest a selective cognitive impairment at the 0.3 and $1.0 \mathrm{mg} / \mathrm{kg}$ doses of CP101,606.

\section{DISCUSSION}

The present studies are the first to demonstrate cognitive impairment in nonhuman primates after administration of NR2B NAMs. The results of these studies clearly show that treatment with a selective NR2B NAM can impair pattern recognition memory (list-DMS) as well as learning and memory of associations between stimuli and location (vsPAL) in cynomolgus monkeys. The use of the leastdifficult trials as procedural controls (eg, 2-s delay in listDMS or two stimuli condition in vsPAL) allows for the separation of impairment in the cognitive domain of the task from other factors affecting performance such as the ability to: (a) remember the rules of the task, (b) perform the motor movements necessary, (c) encode stimuli into working or short-term memory, and (d) accurately recognize the stimuli (or stimulus/location pairing). In addition, factors influencing motivation and psychomotor effects are detected on measures such as percent task completion and response latency. For both CP101,606 and the novel agent, BMT-108908, a delay- or difficulty-dependent impairment was observed at doses not affecting task completion or latency to respond. The results from both tasks are consistent with selective impairment in longer-term memory, and to a lesser extent, learning (vsPAL task) after acute NR2B NAM treatment.

Interestingly, NR2B blockade of the dlPFC neurons studied by Wang et al (2013) is not likely to be responsible for the deficits in DMS performance in the current studies. Lesions of medial but not dorsolateral PFC have been shown to impair DMS performance (Bachevalier and Mishkin, 1986; Passingham, 1975). Patients with frontal lobe lesions were not impaired on the CANTAB DMS task, although frontal patients were impaired on the vsPAL (Owen et al, 1995). Therefore, dlPFC neurons, such as those studied by Wang et al, (2013) may be involved in the spatial memory components of vsPAL; however, the DMS impairment implicates regions outside of the dlPFC. Performance in both vsPAL and DMS tasks is similarly sensitive to function in temporal cortical and hippocampal structures, as well as medial PFC structures, suggesting their involvement in the cognitive impairment observed in the present studies. Additional studies would be needed to provide further information on the relative contribution of different brain

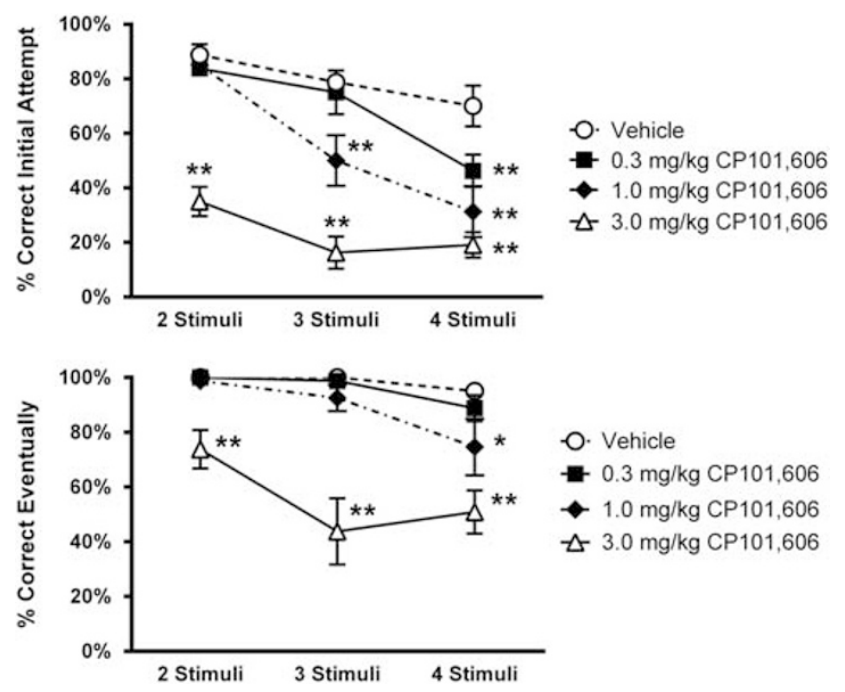

Figure 5 Effect of acute treatment with CPI0I,606 on accuracy in the vsPAL procedure $(N=8)$. Top panel: accuracy on the initial attempt. Bottom panel: eventual accuracy (initial+ five possible repeats). Both panels: $x$ axis is difficulty of trial in terms of number of stumuli. * and *** indicate that performance differs from performance after vehicle at the $p<0.05$ and $p<0.0$ I levels, respectively.

areas to the cognitive impairment seen after NR2B NAM administration.

In healthy human subjects, the ability of ketamine to produce cognitive impairment is well documented. To examine the translational utility of the nonhuman primate CANTAB list-DMS task we also examined the effect of acute ketamine treatment and measured the plasma ketamine concentrations that were achieved after dosing. The ketamine-induced memory impairment in the list-DMS procedure is consistent with previous reports in ketaminetreated monkeys performing the standard CANTAB DMS task (Taffe et al, 2002a). Furthermore, our results are consistent with the dose-dependent impairment of DMS task performance reported in healthy male subjects after i.v. ketamine infusion (Newcomer et al, 1999). In the latter study, the steady state plasma ketamine concentration associated with impaired DMS performance in humans was $90 \mathrm{ng} / \mathrm{ml}(379 \mathrm{nM})$ and is within the exposure range measured over the testing period at the minimum effective dose $(0.56 \mathrm{mg} / \mathrm{kg})$ in cynomolgus monkeys (average plasma concentration $15-60 \mathrm{~min}$ post dose $=595-342 \mathrm{nM}$ ). Indeed, the range of plasma ketamine concentrations associated with memory impairment and impairment on frontal cortical tasks (eg, Wisconsin Card Sort Task) in humans is overlapping with the exposures reported in the present studies (543 nM-1102 nM, (Morgan et al, 2004); $841 \mathrm{nM}$ (Krystal et al, 1998); 513-618 nM, (Hetem et al, 2000)). These results suggest that, at least for ketamine, there is a good correlation between exposure/effect relationships in monkeys and humans.

Although CP101,606 has been administered to humans, the relationship between exposure and cognitive impairment in humans is not well understood, in part because the literature reports of cognitive impairment are largely descriptive. In addition, CP101,606 is metabolized by CYP2D6, a cytochrome P450 enzyme that is highly polymorphic, leading to variable plasma pharmacokinetics 
Table 4 Summary of Details from RM ANOVAs for the Effects of CP I01,606 on vsPAL Performance

\begin{tabular}{|c|c|c|c|}
\hline & Difficulty & Treatment & Interaction \\
\hline \multicolumn{4}{|l|}{ Initial accuracy } \\
\hline $\begin{array}{l}\text { Two-way RM-ANOVA } \\
\text { CPI01,606 PAL }\end{array}$ & $F_{2,14}=43.8 ; p<0.0001$ & $F_{3,21}=41.6 ; p<0.0001$ & $F_{6,42}=5.1 ; p=0.0005$ \\
\hline \multicolumn{4}{|l|}{ Eventual accuracy } \\
\hline $\begin{array}{l}\text { Two-way RM-ANOVA } \\
\text { CPI0I,606 PAL }\end{array}$ & \multicolumn{2}{|c|}{ Response latency } & $\%$ Task completion \\
\hline $\begin{array}{l}\text { One-way RM-ANOVA } \\
\text { CPI0I,606 PAL }\end{array}$ & \multicolumn{2}{|c|}{$F_{3,21}=15.2 ; p<0.0001$} & $F_{3,21}=5.9 ; p=0.004$ \\
\hline
\end{tabular}

Table 5 Group Means for vsPAL Overall Latency to Respond and \% Task Completion

\begin{tabular}{lcc}
\hline CPI0I,606 & $\begin{array}{c}\text { Overall response latency } \\
\text { (ms) (SEM) }\end{array}$ & $\begin{array}{c}\text { \% Task completion } \\
\text { (SEM) }\end{array}$ \\
\hline Vehicle & $1664.8(111.4)$ & $99.5 \%(0.4 \%)$ \\
$0.3 \mathrm{mg} / \mathrm{kg}$ & $1709.6(171.8)$ & $97.3 \%(1.6 \%)$ \\
$1.0 \mathrm{mg} / \mathrm{kg}$ & $1734.7(161.3)$ & $92.4 \%(5.2 \%)$ \\
$3.0 \mathrm{mg} / \mathrm{kg}$ & $2322.8(232.4)^{\mathrm{a}}$ & $80.0 \%(6.4 \%)^{\mathrm{a}}$ \\
\hline
\end{tabular}

andicates performance differed from vehicle at $p<0.01$ level using HolmSidak test.

in subjects who extensively or poorly metabolize the drug (Johnson et al, 2003). Nevertheless, retrograde amnesia and impairment of delayed word recall was reported in healthy humans treated with a $2 \mathrm{~h}$ IV infusion of CP101,606 (Merchant et al, 1999). Amnesia was also reported in healthy subjects receiving an i.v. bolus/infusion administration lasting $72 \mathrm{~h}$ (Merchant et al, 1999). Although plasma drug concentrations were not reported for the $2 \mathrm{~h}$ infusion, the $72 \mathrm{~h}$ infusion paradigm achieved levels of 1466 and $3848 \mathrm{nM}$ in extensive and poor metabolizers, respectively. In the current study, plasma CP101,606 concentrations for impairment of list-DMS performance ranged from 471 to $6423 \mathrm{nM}$ across the effective dose range $(1.0-10 \mathrm{mg} / \mathrm{kg} ; 30 \mathrm{~min}$ post dose ie, at the initiation of testing). Thus, the effect/exposure relationship of CP101,606 in cynomolgus monkeys appears similar to the limited information available from human testing. Interestingly, performance of the nonhuman primate vsPAL appears to be even more sensitive to CP101,606 with a modest but significant impairment detected at the most difficult condition after treatment with $0.3 \mathrm{mg} / \mathrm{kg}$. These results highlight the potential for NR2B NAMs to impair cognition in humans and suggest that well-standardized testing, using tools such as the CANTAB battery, should be incorporated into early clinical testing of novel agents.

These studies are also the first preclinical studies published comparing the cognitive effects of 'low trapping' vs 'high trapping' NMDA antagonists. Preclinical studies have shown that AZD6765 is a modest potency, voltage-dependent, nonselective, NMDA receptor channel blocker (Sanacora et al, 2014). Although this profile is similar to ketamine, the 'low trapping' effects seen in electrophysiology assays are hypothesized to better preserve use-dependent block under conditions of normal synaptic transmission, leading to improved tolerability (Mealing et al, 1999). Indeed, preliminary studies evaluating the antidepressant potential of i.v. infusions of AZD6765 appear consistent with this hypothesis, as AZD6765 showed low potential for dissociative effects, psychotomimetic effects, or cognitive impairment at doses of 100 and $150 \mathrm{mg}$ in patients (Sanacora et al, 2014; Zarate et al, 2013). In the present studies acute treatment with AZD6765 did not impair list-DMS performance at doses of 1 and $3 \mathrm{mg} /$ $\mathrm{kg}$, achieving plasma drug concentrations of $1 \mu \mathrm{M}-656 \mathrm{nM}$ and 3.7-4.2 $\mu \mathrm{M}$, respectively, during the testing period. Although impaired performance accuracy was observed at the highest dose tested, a marked reduction in task completion was also seen, indicating a more general impairment in performance. Indeed we have observed severe emesis in monkeys treated with $10 \mathrm{mg} / \mathrm{kg}$ AZD6765, indicating poor tolerability at higher doses. Although limited plasma exposure information is available in humans, i.v. infusion of $150 / 160 \mathrm{mg}$ AZD6765 is reported to achieve concentrations of $\sim 6 \mu \mathrm{M}$, after a $1 \mathrm{~h}$ infusion (Zarate et al, 2013), similar to levels achieved in these studies.

The ability of NR2B NAMs to impair cognition in nonhuman primates raises the important question as to whether antidepressant efficacy can be dissociated from cognitive effects for this approach. Although this remains to be resolved, it is important to note the temporal disconnect between the transient, short lasting cognitive impairment, which is tightly coupled to plasma exposure and antidepressant effects, which emerge slowly and persist beyond the elimination of ketamine or CP101,606 in humans. Furthermore, although deficient synaptic plasticity (LTP) is thought to underlie cognitive impairment, the delayed antidepressant effect is thought to be driven by enhanced synaptic plasticity in key brain regions implicated in MDD (Duman et al, 2012). In particular, in rodent models of chronic stress, ketamine and NR2B NAMs have been shown to activate the BDNFmTOR signaling pathways, leading to increased translation of synaptic proteins, enhanced synaptogenesis, and alleviation of depression-related behaviors (Duman et al, 2012; Li et al, 2010, 2011). Indeed, using hippocampal LTP as a measure of plasticity it was recently reported that LTP is robustly enhanced in slice preparations taken from animals dosed $24 \mathrm{~h}$ previously with i.v. ketamine or NR2B NAMs 
(Graef et al, 2015). This effect contrasts sharply with the LTP impairment seen following direct application of NMDA antagonists to hippocampal slices (Shipton and Paulsen, 2014). Importantly, although separation of the antidepressant from cognitive effects may ultimately not be achievable, transient cognitive impairment is likely manageable in patients treated with i.v. agents and should not preclude their development. Thus, i.v. infusions will require administration in a clinical setting where patients can be monitored until any transient cognitive issues have resolved. However, the potential for prolonged cognitive impairment following oral NR2B NAMs is of concern especially for agents with long pharmacokinetic half lives. These observations highlight the importance of further studies to investigate tolerance to repeated dosing of NR2B NAMs and the monitoring of cognitive impairment in the clinical development of these agents.

\section{FUNDING AND DISCLOSURE}

This work was funded by Bristol-Myers Squibb, and all authors except for Fu-Ni Cometa and Rudolf Cardinal were BMS employees during their contribution to this project. In the prior three years Fu-ni Cometa has been employed by Aerotek Scientific (during these studies) and Manpower. Dr Cardinal was compensated by BMS for his role in the development of the novel version of the nonhuman primateCANTAB delayed-match-to-sample procedure and software used in these studies. In addition, Dr Cardinal has received salary from the University of Cambridge as a full-time employee; payments for teaching and clinical work from Cambridgeshire and Peterborough NHS Foundation Trust; royalties from software sales via the University of Cambridge; consultancy fees from Campden Instruments Ltd; royalties from books from Taylor Francis Group and Cambridge University Press; a lecture fee from the Neuroscience Education Institute; and payment for clinical services from UK government agencies.

\section{REFERENCES}

Abdallah CG, Averill LA, Krystal JH (2015). Ketamine as a promising prototype for a new generation of rapid-acting antidepressants. Ann N Y Acad Sci 1344: 66-77.

Bachevalier J, Mishkin M (1986). Visual recognition impairment follows ventromedial but not dorsolateral prefrontal lesions in monkeys. Behav Brain Res 20: 249-261.

Buccafusco JJ, Terry AV Jr. (2009). A reversible model of the cognitive impairment associated with schizophrenia in monkeys: potential therapeutic effects of two nicotinic acetylcholine receptor agonists. Biochem Pharmacol 78: 852-862.

Cardinal RN, Aitken MR (2010). Whisker: a client-server highperformance multimedia research control system. Behav Res Methods 42: 1059-1071.

Coyle CM, Laws KR (2015). The use of ketamine as an antidepressant: a systematic review and meta-analysis. Hum Psychopharmacol 30: 152-163.

Duman RS, Li N, Liu RJ, Duric V, Aghajanian G (2012). Signaling pathways underlying the rapid antidepressant actions of ketamine. Neuropharmacology 62: 35-41.

Graef JD, Newberry K, Newton A, Pieschl R, Shields E, Luan FN et al (2015). Effect of acute NR2B antagonist treatment on longterm potentiation in the rat hippocampus. Brain Res 1609: 31-39.
Guscott MR, Clarke HF, Murray F, Grimwood S, Bristow LJ, Hutson PH (2003). The effect of (+/-)-CP-101,606, an NMDA receptor NR2B subunit selective antagonist, in the Morris watermaze. Eur J Pharmacol 476: 193-199.

Hanson JE, Weber M, Meilandt WJ, Wu T, Luu T, Deng L et al (2013). GluN2B antagonism affects interneurons and leads to immediate and persistent changes in synaptic plasticity, oscillations, and behavior. Neuropsychopharmacology 38: 1221-1233.

Hetem LA, Danion JM, Diemunsch P, Brandt C (2000). Effect of a subanesthetic dose of ketamine on memory and conscious awareness in healthy volunteers. Psychopharmacology 152: 283-288.

Higgins GA, Ballard TM, Enderlin M, Haman M, Kemp JA (2005). Evidence for improved performance in cognitive tasks following selective NR2B NMDA receptor antagonist pre-treatment in the rat. Psychopharmacology 179: 85-98.

Higgins GA, Ballard TM, Huwyler J, Kemp JA, Gill R (2003). Evaluation of the NR2B-selective NMDA receptor antagonist Ro 63-1908 on rodent behaviour: evidence for an involvement of NR2B NMDA receptors in response inhibition. Neuropharmacology 44: 324-341.

Johnson K, Shah A, Jaw-Tsai S, Baxter J, Prakash C (2003). Metabolism, pharmacokinetics, and excretion of a highly selective $\mathrm{N}$-methyl-D-aspartate receptor antagonist, traxoprodil, in human cytochrome P450 2D6 extensive and poor metabolizers. Drug Metab Dispos 31: 76-87.

Kos T, Nikiforuk A, Rafa D, Popik P (2011). The effects of NMDA receptor antagonists on attentional set-shifting task performance in mice. Psychopharmacology 214: 911-921.

Krystal JH, Karper LP, Bennett A, D'Souza DC, Abi-Dargham A, Morrissey $\mathrm{K}$ et al (1998). Interactive effects of subanesthetic ketamine and subhypnotic lorazepam in humans. Psychopharmacology 135: 213-229.

Li N, Lee B, Liu RJ, Banasr M, Dwyer JM, Iwata M et al (2010). mTOR-dependent synapse formation underlies the rapid antidepressant effects of NMDA antagonists. Science 329: 959-964.

Li N, Liu RJ, Dwyer JM, Banasr M, Lee B, Son H et al (2011). Glutamate $\mathrm{N}$-methyl-D-aspartate receptor antagonists rapidly reverse behavioral and synaptic deficits caused by chronic stress exposure. Biol Psychiatry 69: 754-761.

Mealing GA, Lanthorn TH, Murray CL, Small DL, Morley P (1999). Differences in degree of trapping of low-affinity uncompetitive $\mathrm{N}$-methyl-D-aspartic acid receptor antagonists with similar kinetics of block. J Pharmacol Exp Ther 288: 204-210.

Menniti FS, Shah AK, Williams SA, Wilner KD, White WF, Chenard BL (1998). CP-101,606: an NR2B-selective NMDA receptor antagonist. CNS Drug Rev 4: 307-322.

Merchant RE, Bullock MR, Carmack CA, Shah AK, Wilner KD, Ko $\mathrm{G}$ et al (1999). A double-blind, placebo-controlled study of the safety, tolerability and pharmacokinetics of CP-101,606 in patients with a mild or moderate traumatic brain injury. Ann $N$ $Y$ Acad Sci 890: 42-50.

Morgan CJ, Curran HV (2006). Acute and chronic effects of ketamine upon human memory: a review. Psychopharmacology 188: 408-424.

Morgan CJ, Mofeez A, Brandner B, Bromley L, Curran HV (2004). Acute effects of ketamine on memory systems and psychotic symptoms in healthy volunteers. Neuropsychopharmacology 29: 208-218.

Neill JC, Barnes S, Cook S, Grayson B, Idris NF, McLean SL et al (2010). Animal models of cognitive dysfunction and negative symptoms of schizophrenia: focus on NMDA receptor antagonism. Pharmacol Ther 128: 419-432.

Newcomer JW, Farber NB, Jevtovic-Todorovic V, Selke G, Melson AK, Hershey T et al (1999). Ketamine-induced NMDA receptor hypofunction as a model of memory impairment and psychosis. Neuropsychopharmacology 20: 106-118. 
Newcomer JW, Krystal JH (2001). NMDA receptor regulation of memory and behavior in humans. Hippocampus 11: 529-542.

NRC (2011). National Research Council. Guide for the Care and Use of Laboratory Animals. 8th edn. National Academies Press: Washington, DC, USA.

Nutt JG, Gunzler SA, Kirchhoff T, Hogarth P, Weaver JL, Krams M et al (2008). Effects of a NR2B selective NMDA glutamate antagonist, CP-101,606, on dyskinesia and Parkinsonism. Mov Disord 23: 1860-1866.

Okiyama K, Smith DH, White WF, Richter K, McIntosh TK (1997). Effects of the novel NMDA antagonists CP-98,113, CP-101,581 and CP-101,606 on cognitive function and regional cerebral edema following experimental brain injury in the rat. J Neurotrauma 14: 211-222.

Owen AM, Sahakian BJ, Semple J, Polkey CE, Robbins TW (1995). Visuo-spatial short-term recognition memory and learning after temporal lobe excisions, frontal lobe excisions or amygdalohippocampectomy in man. Neuropsychologia 33: 1-24.

Passingham R (1975). Delayed matching after selective prefrontal lesions in monkeys (Macaca mulatta). Brain Res 92: 89-102.

Preskorn SH, Baker B, Kolluri S, Menniti FS, Krams M, Landen JW (2008). An innovative design to establish proof of concept of the antidepressant effects of the NR2B subunit selective N-methyl-Daspartate antagonist, CP-101,606, in patients with treatmentrefractory major depressive disorder. J Clin Psychopharmacol 28: 631-637.

Roberts BM, Seymour PA, Schmidt CJ, Williams GV, Castner SA (2010). Amelioration of ketamine-induced working memory deficits by dopamine D1 receptor agonists. Psychopharmacology 210: $407-418$.

Sanacora G, Schatzberg AF (2015). Ketamine: promising path or false prophecy in the development of novel therapeutics for mood disorders? Neuropsychopharmacology 40: 259-267.
Sanacora G, Smith MA, Pathak S, Su HL, Boeijinga PH, McCarthy DJ et al (2014). Lanicemine: a low-trapping NMDA channel blocker produces sustained antidepressant efficacy with minimal psychotomimetic adverse effects. Mol Psychiatry 19: 978-985.

Shipton OA, Paulsen O (2014). GluN2A and GluN2B subunitcontaining NMDA receptors in hippocampal plasticity. Philos Trans R SocLond B Biol Sci 369: 20130163.

Smith JW, Gastambide F, Gilmour G, Dix S, Foss J, Lloyd K et al (2011). A comparison of the effects of ketamine and phencyclidine with other antagonists of the NMDA receptor in rodent assays of attention and working memory. Psychopharmacology 217: 255-269.

Taffe MA, Davis SA, Gutierrez T, Gold LH (2002a). Ketamine impairs multiple cognitive domains in rhesus monkeys. Drug Alcohol Depend 68: 175-187.

Taffe MA, Weed MR, Gutierrez T, Davis SA, Gold LH (2002b). Differential muscarinic and NMDA contributions to visuo-spatial paired-associate learning in rhesus monkeys. Psychopharmacology 160: 253-262.

Wang M, Yang Y, Wang CJ, Gamo NJ, Jin LE, Mazer JA et al (2013). NMDA receptors subserve persistent neuronal firing during working memory in dorsolateral prefrontal cortex. Neuron 77: 736-749.

Weed MR, Taffe MA, Polis I, Roberts AC, Robbins TW, Koob GF et al (1999). Performance norms for a rhesus monkey neuropsychological testing battery: acquisition and long-term performance. Brain Res Cogn Brain Res 8: 185-201.

Zarate CA Jr., Mathews D, Ibrahim L, Chaves JF, Marquardt C, Ukoh I et al (2013). A randomized trial of a low-trapping nonselective $\mathrm{N}$-methyl-D-aspartate channel blocker in major depression. Biol Psychiatry 74: 257-264.

Zhang XH, Liu SS, Yi F, Zhuo M, Li BM (2013). Delay-dependent impairment of spatial working memory with inhibition of NR2Bcontaining NMDA receptors in hippocampal CA1 region of rats. Mol Brain 6: 13.

Supplementary Information accompanies the paper on the Neuropsychopharmacology website (http://www.nature.com/npp) 